\title{
Critical Care and Emergency Medicine
}

Open Access

CASE REPORT

\section{Thinking Outside the "Box": Severe Intra-Abdominal Bleeding Due to Liver Injury Post CPR}

\section{Catsim Fassassi and Ronald Simon*}

Maimonides Medical Center, USA

*Corresponding author: Ronald Simon, MD, Director of Acute Care Surgery, Division of Trauma, Maimonides Medical Center, 4901-A Fort Hamilton Parkway, Lower Level, Brooklyn, New York 11219, USA, Tel: 718-635-1409, Fax: 718-635-6044

\section{Background}

Chest compressions are the most important element of cardiopulmonary resuscitation (CPR) [1]. When appropriate chest compressions are performed, coronary perfusion pressure is optimized with the greatest chance for the return of spontaneous circulation (ROSC). However, CPR is not without complications. Fractured ribs and sternums, aspiration pneumonia, and mediastinal hemorrhage have been well documented [2-4]. Less well-described are intra-abdominal complications, including stomach, spleen and liver injuries [5]. Significant liver injuries, though rare, when misdiagnosed or addressed late in the patient's hospital course, may lead to poor outcomes $[3,4,6]$. We report a case of hepatic injury suggested by FAST and confirmed by CT after resuscitation from cardiac arrest. Based on this case, we propose routine Focused Abdominal Ultrasound for Trauma (FAST) screening in all post-resuscitative patients to rule out CPR induced liver injury, before they present with sudden hemodynamic instability or dropping hematocrits.

\section{Case}

35-year-old woman with a past psychiatric history and alcohol abuse was found unresponsive on the bathroom floor by her boyfriend. EMS was called, and chest compressions were commenced by the boyfriend while awaiting EMS arrival. EMS found the patient to be in PEA and continued CPR on scene and during transport. ROSC was achieved while en route to the hospital. On arrival to the ED she was initially normotensive but quickly decompensated requiring aggressive fluid

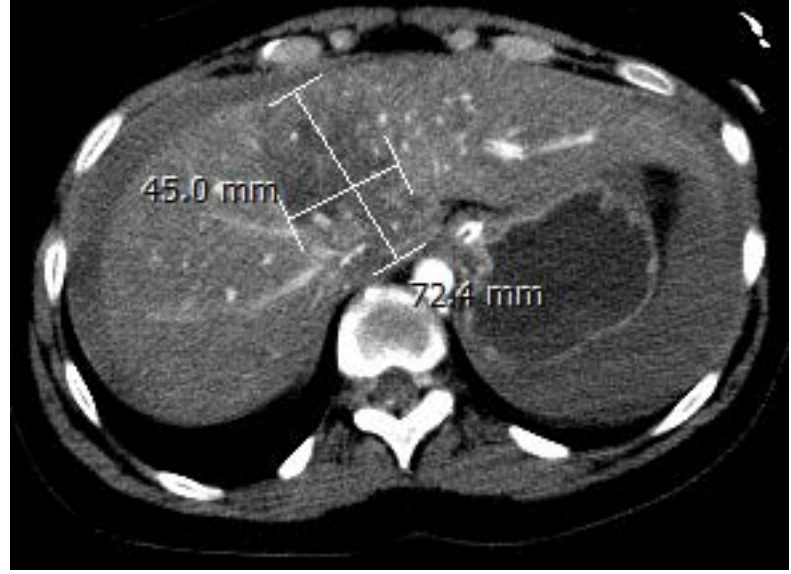

Figure 1: Liver laceration.

resuscitation and vasopressors. FAST was performed by ED staff, revealing hemoperitoneum. A CT scan and surgical consult were obtained. CT showed a grade 4 liver laceration which occurred in the plane between the sternum and the spine suggesting a compression injury from the CPR (see Figure 1). As there was no evidence of active extravasation or a pseudoaneurysm, surgery determined that no further intervention was needed and to following hematocrits for $24 \mathrm{hrs}$. She was admitted to the ICU for ventilator and vasopressor management. Her neurological function remained severely depressed and prognosis was deemed poor. She was made DNR/ DNI by family and terminally extubated.

\section{Discussion}

Liver injuries are the most common [5] intra-abdominal complications following CPR, with an approxi- 
mate incidence of 0.6 to $3 \%[2,4]$. The left liver lobe is mainly affected due to its proximity the sternum [2]. Antiplatelet, antithrombotic and thrombolytic treatments especially in patients with suspected myocardial infarction or pulmonary embolism have been cited as contributing factors for intra-abdominal hemorrhage [5-7]. Liver injury and intra-abdominal bleeding can either be missed or diagnosed late (hospital day 2 or later) $[3,5]$. Therefore the true incidence may be underreported, particularly in unconscious patients or patients were support is felt to be futile and full evaluations are felt to be irrelevant. Hemodynamic instability after successful ROSC is often attributed to cardiac dysfunction. Bleeding from an intra-abdominal source is often not considered. Transporting a hemodynamically unstable, post arrest patient for an abdominal CT scan is not without its own risks. The difficulty in making the diagnosis in a hemodynamically labile patient with altered mental function supports surveillance. By employing FAST screening in post-resuscitative patients, particularly those who instability cannot be easily attributed to cardiac dysfunction, display an acute drop in hemoglobin and/ or abdominal distention should raise suspicion for intra-abdominal injury [8]. Limitations of ultrasound include operator dependence, patient related factors such as obesity, and failure to detect fluid volumes less than $500 \mathrm{cc}$ [9]. Surgical intervention has been suggested to achieve bleeding control; however reported mortality is high [6]. A minimally invasive treatment strategy that includes angiographic embolization and percutaneous evacuation of intra-abdominal blood has been reported with favorable outcomes [10].

\section{Conclusion}

An intra-abdominal injury status post CPR, is a rare, but a potentially life-threatening complication. It is often missed or diagnosed late due to low index of suspicion and the assumption that hemodynamic instability is secondary to a cardiac etiology. Vigilance is required, and we suggest routine screening using FAST to evaluate post-resuscitative patients for intra-abdominal fluid, particularly those displaying labile hemodynamics, acu- te drop in hemoglobin or abdominal distention. If fluid is found, we recommend CT scan with contrast to assess for CPR induced hepatic injury and assess whether further intervention, such as angiographic embolization, is indicated.

\section{References}

1. Gallagher EJ, Lombardi G, Gennis P (1995) Effectiveness of bystander cardiopulmonary resuscitation and survival following out-of-hospital cardiac arrest. JAMA 274: 19221925.

2. Buschmann CT, Tsokos M (2009) Frequent and rare complications of resuscitation attempts. Intensive Care Med 35: 397-404.

3. Zahn G, Hauck M, Pearson DA, Green JM, Heffner AC (2015) Major hemorrhage from hepatic laceration after cardiopulmonary resuscitation. Am J Emerg Med 33: 991.e3991.e4.

4. Beydilli H, Balci Y, Erbas M, Acar E, Isik S, et al. (2016) Liver laceration related to cardiopulmonary resuscitation. Turk J Emerg Med 16: 77-79.

5. Joseph JR, Freundlich RE, Abir M (2016) Ruptured subcapsular liver hematoma following mechanically-assisted cardiopulmonary resuscitation. BMJ Case Rep.

6. Meron G, Kurkciyan I, Sterz F, Susani M, Domanovits H, et al. (2007) Cardiopulmonary resuscitation-associated major liver injury. Resuscitation 75: 445-453.

7. Biswas S, Alpert A, Lyon M, Kaufmann CR (2017) Cardiopulmonary resuscitation complicated by traumatic hepatic laceration: A case report and review of literature. J Med Case Report 8: 93-97.

8. Nashiki H, Miyate $\mathrm{Y}$, Terui $\mathrm{Y}$, Otani M (2017) Focused assessment with sonography for trauma (FAST) identifies liver injury following cardiopulmonary resuscitation. BMJ Case Rep.

9. Sheng AY, Dalziel P, Liteplo AS, Peter Fagenholz, Vicki E Noble (2013) Focused assessment with sonography in trauma and abdominal computed tomography utilization in adult trauma patients: Trends over the last decade. Emerg Med Int 2013: 678380

10. Næss PA, Engeseth K, Grøtta O, Andersen G $\varnothing$, Gaarder C (2016) Minimal invasive treatment of life-threatening bleeding caused by cardiopulmonary resuscitation-associated liver injury: A case report. J Med Case Rep 10: 132. 